\title{
Oscillatory behavior of the tunnel magnetoresistance due to thickness variations in Ta|CoFe|MgO magnetic tunnel junctions: A first-principles study
}

\author{
K. Sankaran,,${ }^{1,}$ J. Swerts, ${ }^{1}$ S. Couet, ${ }^{1}$ K. Stokbro, ${ }^{2}$ and G. Pourtois ${ }^{1,3}$ \\ ${ }^{1}$ imec, Kapeldreef 75, 3000 Leuven, Belgium \\ ${ }^{2}$ QuantumWise A/S, Fruebjergvej 3 Box 4, 2100 Copenhagen, Denmark \\ ${ }^{3}$ PLASMANT, Department of Chemistry, University of Antwerp, 2610 Antwerp, Belgium \\ (Received 4 November 2015; revised manuscript received 17 March 2016; published 20 September 2016)

\begin{abstract}
To investigate the impact of both the CoFe ferromagnetic layer thickness and the capping paramagnetic layer on the tunnel magnetoresistance (TMR), we performed first-principles simulations on epitaxial magnetic tunnel junctions contacted with either CoFe or Ta paramagnetic capping layers. We observed a strong oscillation of the TMR amplitude with respect to the thickness of the ferromagnetic layer. The TMR is found to be amplified whenever the $\mathrm{MgO}$ spin tunnel barrier is thickened. Quantization of the electronic structure of the ferromagnetic layers is found to be at the origin of this oscillatory behavior. Metals such as Ta contacting the magnetic layer are found to enhance the amplitude of the oscillations due to the occurrence of an interface dipole. The latter drives the band alignment and tunes the nature of the spin channels that are active during the tunneling process. Subsequently, the regular transmission spin channels are modulated in the magnetic tunnel junction stack and other complex ones are being activated.
\end{abstract}

DOI: 10.1103/PhysRevB.94.094424

\section{INTRODUCTION}

Magnetic tunnel junctions (MTJs) are based on a differential magnetoresistance effect. The resistance of an MTJ stack is modulated whenever the magnetic moments of the two ferromagnetic electrodes, separated by an insulating tunnel barrier, are switched between a parallel (P) and an antiparallel (AP) state [1]. This effect has attracted the attention of the engineering community and opened the door to the development of a new class of low-power solid-state magnetic memories, generally being referred to as magnetoresistive random access memory (MRAM) [2]. The difference in resistance arises from the half-metallic character of the electrodes in which only one spin character is present at the Fermi level. The insulating barrier filters the electron wave functions based on their symmetries with different decay rates inside the barrier. This leads to substantial changes in the magnetoresistance, even for electrodes that do not have complete half-metallicity [3]. This feature, known as spin-dependent tunneling, leads to different conductances when the two electrodes have different spin orientations, being either parallel $\left(G_{\mathrm{P}}\right)$ or antiparallel $\left(G_{\mathrm{AP}}\right)$. This change in conductance is defined by the tunnel magnetoresistance (TMR) as $\mathrm{TMR}=\frac{G_{\mathrm{P}}-G_{\mathrm{AP}}}{G_{\mathrm{AP}}}[\mathrm{Eq}$. (1)]. The TMR is one of the key properties of the MTJ and is being employed as a reference criterion to rank the performance of MTJ stacks for memory applications. The enhancement of the TMR is a driving factor for the development of high-performance nanometer-scaled magnetic devices.

The earliest attempt to build MTJ stacks using an amorphous $\mathrm{Al}_{2} \mathrm{O}_{3}$ insulating barrier sandwiched with two $\mathrm{Fe}$ ferromagnetic layers has met with moderate success [4]. The resulting TMR at room temperature was too low to allow the development of high-performance devices. More recent studies, using an epitaxial crystalline $\mathrm{MgO}$ barrier grown on bcc Fe electrodes, have shown that a high TMR on the order

\footnotetext{
*sankaran@imec.be
}

of few hundred percent was achievable [5,6]. In that system, the high TMR value originates from the cubic symmetry of the crystalline $\mathrm{MgO}$ barrier that selectively filters the spin tunneling based on the symmetry of the wave function. In the $\mathrm{MgO}$ barrier, the electron wave functions with a $\Delta_{1}\left(s, p_{z}, d_{z}^{2}\right)$ symmetry decay more slowly than those with a $\Delta_{2}\left(d_{x}^{2}-d_{y}^{2}\right)$ and $\Delta_{5}\left(p_{x z}, p_{y z}, d_{x z}, d_{y z}\right)$ symmetry [7]. This is due to the availability of the majority-spin channel $\Delta_{1}$ and $\Delta_{5}$ bands at the Fermi level along the [001] direction in the bcc $\mathrm{Fe}$ layer, whereas in the minority-spin channel, only the $\Delta_{2}$ and $\Delta_{5}$ states cross the Fermi level. Other degenerated bands, such as the $\Delta_{2}^{\prime}$ one, are similarly present at the Fermi level and decay rapidly in the $\mathrm{MgO}$ barrier. Consequently, a high TMR ratio is achievable. Recent theoretical works based on first-principles studies have shown that even higher TMR values can be achieved in epitaxial MTJ stacks with either bcc $\mathrm{Co}(\mathrm{Co}|\mathrm{MgO}| \mathrm{Co})$ or $\mathrm{Fe}_{0.5} \mathrm{Co}_{0.5}\left(\mathrm{Fe}_{0.5} \mathrm{Co}_{0.5}|\mathrm{MgO}| \mathrm{Fe}_{0.5} \mathrm{Co}_{0.5}\right)$ as ferromagnetic layers $[7,8]$. The enhancement in tunneling of these MTJs is attributed to a direct contact between Co and $\mathrm{O}$ atoms at the interface with the insulating layer [8]. This finding has inspired the scientific and engineering communities to focus on FeCo alloys as ferromagnetic electrodes to maximize the TMR [9]. In practice, numerous requirements have to be met to build high-density, high-performance spin-transfer torque (STT) magnetic memories using MTJs. Among these, the presence of a large perpendicular magnetic anisotropy (PMA) in ferromagnetic thin layers (from 1 to $2 \mathrm{~nm}$ ) with low Gilbert damping is particularly important for memory retention and low-power operability [10]. It has been reported that, when Co is residing between the ferromagnetic electrodes and the barrier, the MTJ stack adopts a low PMA, which is detrimental for the reliability of the memory [11]. Therefore $\mathrm{CoFe}$ is the material of choice as a best-value tradeoff between PMA and TMR [10]. Although MTJs with CoFe|MgO|CoFe have been extensively studied during the past years, the investigation of the dependence of the CoFe polarizer thickness on the TMR is uncompleted. Furthermore, most theoretical works focus on the primary $\mathrm{CoFe}|\mathrm{MgO}| \mathrm{CoFe}$ interface and 


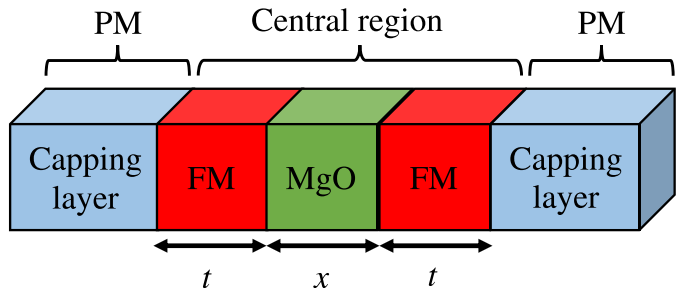

FIG. 1. Schematic illustration of a MTJ stack composed of ferromagnetic (FM) CoFe layers (with different thicknesses $t$ ) and an insulating $\mathrm{MgO}$ barrier (with different thicknesses $x$ ) with paramagnetic (PM) capping layers (with fixed thickness).

neglect the possible impact of the contacting electrodes on the device performance (see Fig. 1). With this paper, we aim to fill this gap.

Some reports $[12,13]$ focused on the quantification of the TMR going from subnanometer- to nanometer-thick crystalline Fe ferromagnetic electrodes. Due to the confinement of the wave functions in the ferromagnetic film, the electronic structure of the metal undergoes changes that are large enough to affect the TMR ratio of a $\mathrm{Fe}|\mathrm{MgO}| \mathrm{Fe}$ MTJ. The effect is much stronger for TMR than for giant magnetoresistance (GMR) in metallic multilayers. Moreover, the amplitude of the TMR oscillations depends on the $\mathrm{MgO}$ barrier thickness. This phenomenon is present despite the introduction of interface disorders between the ferromagnetic layers and the $\mathrm{MgO}$ barrier $[12,13]$. In opposition to these theoretical predictions, it has been reported that these oscillations are actually not observed during experimental measurements of the TMR likely due to the high degree of disorder present in the alloys and at the interfaces of the MTJ stacks [13]. In order to improve the interface quality, thin metallic nonmagnetic capping layers (such as tantalum) on the order of few angstroms are typically grown on the ferromagnetic CoFe layers. The capping layer prevents the crystal texture of other layers (such as the ones of the synthetic antiferromagnet) to be transferred to $\mathrm{CoFe}$. Typically, Ta capping layers are used to encapsulate the MTJ stack [14]. Unfortunately, the introduction of these paramagnetic layers can also impact the switching behavior of the memory $[15,16]$. Understanding both the role of the crystalline CoFe ferromagnetic electrode thickness and the impact of the paramagnetic capping layers could help the scientific and engineering communities in their search for high TMR. In addition, from a modeling perspective, the effect of the capping layers is often neglected whenever the TMR is evaluated for an MTJ stack, in order to minimize the computational burden [13]. The capping layers are often described as the extension of the ferromagnetic electrodes with their magnetic properties being switched off. This is implicitly assuming that the enforced paramagnetic nature of the capping layers does not impact the qualitative outcome of the modeled TMR [13]. Through this publication, we will show using first-principles simulations that the validity of this assumption depends on the nature of the contacting metal. To investigate the impact of the $\mathrm{CoFe}$ ferromagnetic layer thickness on the TMR and to study the role of the contacting Ta layer, we used a perfect epitaxial MTJ stack contacted with either CoFe or Ta paramagnetic capping layers (capping layer $|\mathrm{CoFe}| \mathrm{MgO}|\mathrm{CoFe}|$ capping layer). The capping layers and $\mathrm{CoFe}$ contacting $\mathrm{MgO}$ are chosen to be paramagnetic and ferromagnetic, respectively. We investigated the TMR dependency on the thickness of thin $\mathrm{CoFe}$ ferromagnetic layers [with values ranging from 3 to 17 monolayers (i.e., about $2.3 \mathrm{~nm}$ )] and $\mathrm{MgO}$ barrier [spanning from 3 to 11 monolayers (i.e., about $2.1 \mathrm{~nm}$ )]. We also investigated the role on the TMR of Ta capping layers stressing the impact of the nature and of the atomic density of the contact metal electrode on the TMR.

The paper is organized as follows: First, the methodology is described together with the structural properties of the MTJ stack. We then review the results of the TMR dependence on the $\mathrm{CoFe}$ ferromagnetic film thickness in the presence and absence of capping layers.

\section{METHODOLOGY}

The $\mathrm{CoFe}|\mathrm{MgO}| \mathrm{CoFe}$ junctions are built using the structural parameters reported in the seminal work of Yang et al. [17] on $\mathrm{Fe}|\mathrm{MgO}| \mathrm{Fe}$ MTJs. The relaxed atomic positions and structural parameters reported in Ref. [17] take into account the mismatch in the lateral lattice constant between bulk bcc $\mathrm{Fe}(2.866 \AA)$ [CoFe $(2.857 \AA)]$ and bulk $\mathrm{MgO}(2.977 \AA)$. Because of the small lattice mismatch, the Fe layers expand in the lateral direction, while the $\mathrm{MgO}$ layers slightly shrink to reach a value of $2.93 \AA$ [17]. Using the relaxed atomic positions and the structural parameters of Ref. [17] for the MTJ stacks and selectively substituting Co for Fe, we created a layered crystalline CoFe alloy. Tsai et al. [18] reported that preferentially $\mathrm{Fe}-\mathrm{O}$ bonds are formed at the interface with $\mathrm{MgO}$ during the deposition and postdeposition thermal anneal. Therefore, we created a junction model in which the $\mathrm{CoFe}$ surface contacting the $\mathrm{MgO}$ is terminated by a Fe monolayer. This ensures a high PMA [17]. Note that the bond length between $\mathrm{Fe}$ and $\mathrm{O}$ (about $2.10 \AA$ ) at the interface has been set to match the relaxed lattice and atomic positions reported in Ref. [17]. Finally, we assumed that the Ta capping layer is epitaxially grown on the CoFe. The Ta hence adopts a bcc symmetry similar to CoFe. To minimize the stress in the $\mathrm{Ta}$ layers, the atomic positions of the Ta atoms in contact with $\mathrm{CoFe}$ have been relaxed. The minimization process of the atomic positions of the $\mathrm{Ta}$ is thus conducted while maintaining the $\mathrm{CoFe}$ and $\mathrm{MgO}$ sites fixed. A full relaxation of the atomic position in the stack would alter the topology of the perfect $\mathrm{CoFe}$ and $\mathrm{MgO}$ interface and as such hinder a direct comparison between the $\mathrm{CoFe}|\mathrm{MgO}| \mathrm{CoFe}$ junctions with and without a Ta capping layer. We also neglected all experimentally observed film characteristics such as surface roughness, grain boundaries, and intermixing (or other defects) to ensure computationally tractable calculations. Additional information on the MTJ model for the stacks with the Ta capping layers can be found in the Appendix.

To quantify the spin-transport properties, we used the firstprinciples ATOMISTIX TOOLKIT packages [19], which combine density functional theory (DFT) with nonequilibrium Green's functions (NEGFs). The collinear spin-polarized PerdewBurke-Ernzerhof (PBE) generalized gradient approximation [20] is employed to quantify the exchange-correlation potential. During the self-consistent resolution of the KohnSham equations, the Hamiltonian is forced to converge to 
a tolerance of $10^{-6} \mathrm{Ha}$. Double- $\zeta$ polarized (DZP) basis sets and a mesh cutoff energy of 150 Ry are used for the description of the electron wave function together with a $6 \times 6 k$-point grid to sample the Brillouin zone along the plane parallel to the interface and a $100 \mathrm{k}$-point grid along the perpendicular direction during the self-consistent calculations. This ensures an accurate evaluation of the electrodes' chemical potential and it guarantees the convergence for the energy $\left(10^{-6} \mathrm{eV}\right)$.

The electronic population follows the Fermi-Dirac distribution computed at $300 \mathrm{~K}$. These parameters yield a good balance between the computational time and the accuracy. The conductance for the $\mathrm{P}$ and $\mathrm{AP}\left(G_{\mathrm{P}}\right.$ and $\left.G_{\mathrm{AP}}\right)$ configurations are calculated in the linear response regime. The transport calculations are performed within the elastic limit, in which the transmission probabilities at a given energy are calculated for a specific k-vector $\left(k_{\|}\right)$in the two-dimensional Brillouin zone perpendicular to the transport direction. The ballistic conductance is related to the total transmission at the Fermi energy as given by the Landauer-Büttiker formula: $\mathrm{G}=\frac{e^{2}}{h} \sum T\left(k_{\|}\right)$, where $T\left(k_{\|}\right)$is the transmission probability of the electron with spin at the Fermi energy [19] and $k_{\|}=k_{x y}$ is the Bloch wave vector corresponding to the periodic component of the junction. More details can be found in Ref. [19]. Furthermore, a finer Monkhorst-Pack integration grid of $30 \times 30$ is used to calculate the different conductances in the two-dimensional Brillouin zone perpendicular to the transport direction. Once these conductances are obtained, the TMR ratio is calculated using Eq. (1). Insights into the nature of the active channels in the conductance are gained by resolving the transmission spectrum along the $\mathbf{k}$ points. The NEGF formalism requires defining transmission electrodes (left and right ones) from which the transmission probability of an electron present in a central region is computed [19]. In this work, the central region corresponds to the $\mathrm{CoFe}|\mathrm{MgO}| \mathrm{CoFe} \mathrm{MTJ}$ stack, while the left and right electrodes are the paramagnetic Ta capping layers. In the case of the MTJ stacks without capping layer, the left and right electrodes are CoFe layers that are forced to be paramagnetic. The $\mathrm{CoFe}$ in the scattering region remains, however, ferromagnetic to break the delocalization of the spin wave function in the electrode and to induce its frustration. As a consequence, the presence of an artificial interface between the paramagnetic and the ferromagnetic CoFe impacts the computed TMR as detailed in the next section. The thickness variations used in the $\mathrm{CoFe}$ ferromagnetic and $\mathrm{MgO}$ barrier are hence restricted to only the central region, as schematized in Fig. 1.

\section{RESULTS}

\section{A. Uncapped MTJ stacks}

Figure 2 shows the computed TMR as a function of the $\mathrm{CoFe}(\mathrm{fm})$ layer thickness for different $\mathrm{MgO}$ layers in a $\mathrm{CoFe}(\mathrm{pm})|\mathrm{CoFe}(\mathrm{fm})| \mathrm{MgO}|\mathrm{CoFe}(\mathrm{fm})| \mathrm{CoFe}(\mathrm{pm})$ (pm stands for paramagnetic and fm for ferromagnetic) epitaxial junction in the presence of a paramagnetic $\mathrm{CoFe}(\mathrm{pm})$ capping layer with the same crystal structure and atomic density as the $\mathrm{CoFe}(\mathrm{fm})$ polarizer. The TMR increases with the $\mathrm{MgO}$ barrier thickness, which is an expected trend for epitaxial MTJs due

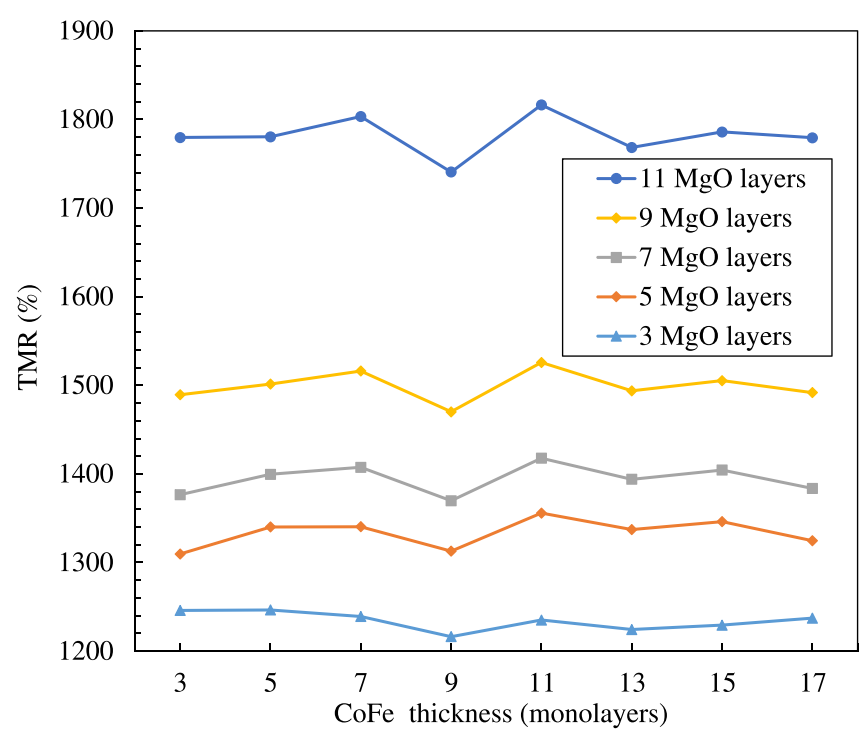

FIG. 2. Evolution of the computed TMR for CoFe(paramagnetic) $\mid \mathrm{CoFe}$ (ferromagnetic) $|\mathrm{MgO}| \mathrm{CoFe}$ (ferromagnetic) $\mid \mathrm{CoFe}$ (paramagnetic) MTJ stack as a function of the ferromagnetic $\mathrm{CoFe}$ thickness for different $\mathrm{MgO}$ barrier thicknesses.

to the efficient filtering of the interfering spin channels in the $\mathrm{MgO}$ barrier. The latter is reviewed in detail by Butler et al. [7]. Secondly, the amplitude of the TMR oscillates for certain thicknesses of the ferromagnetic CoFe. These oscillations originate from the fluctuations in the conductance, which are typically observed for the frustrated/confined spin wave functions that occur between ferromagnetic layers sandwiched by paramagnetic ones, as reported for metallic multilayers [13]. Keeping the material characteristics in the $\mathrm{CoFe}(\mathrm{pm})$ capping layer similar to the $\mathrm{CoFe}(\mathrm{fm})$ polarizer leads to an artificial frustration of the spin in the scattering region (as detailed above). The change in the description of the magnetization of the system is sufficient to frustrate/confine the propagation of the spin wave function. The resulting oscillations, although being ascribed to a pure artifact of the stack simulation, provide useful guidance to reveal the impact on the TMR of the paramagnetic capping layers, as detailed in the following sections. For a thin $\mathrm{MgO}$ (3 monolayers, for instance), the amplitude of the oscillations remains nearly constant for increasing CoFe thicknesses. They, however, become more and more pronounced upon the thickening of the $\mathrm{MgO}$ tunneling barrier. This is in agreement with a previous modeling report of Ref. [13], where the MTJ stacks built from Fe ferromagnetic and $\mathrm{MgO}$ such as $\mathrm{Fe}(\mathrm{pm})|\mathrm{Fe}(\mathrm{fm})| \mathrm{MgO}|\mathrm{Fe}(\mathrm{fm})| \mathrm{Fe}(\mathrm{pm})$ lead to an oscillatory behavior even for thin Fe ferromagnetic layers. This was, however, reported to be possible only due to the presence of a thick $\mathrm{MgO}$ tunnel barrier (from 10 to 24 monolayers). It is interesting to note from Fig. 2 that the variations of the amplitude of the TMR are more pronounced for thick $\mathrm{MgO}$ tunnel barriers (for 9 and 10 monolayers) rather than for thin ones (as is the case for 3 monolayers). The latter shows that thick $\mathrm{MgO}$ tunnel barriers act as an efficient filtering of the interfering spin channel and subsequently boost not only the TMR ratio but also its oscillations. Moreover, further thickening of the $\mathrm{MgO}$ tunnel barrier shows 


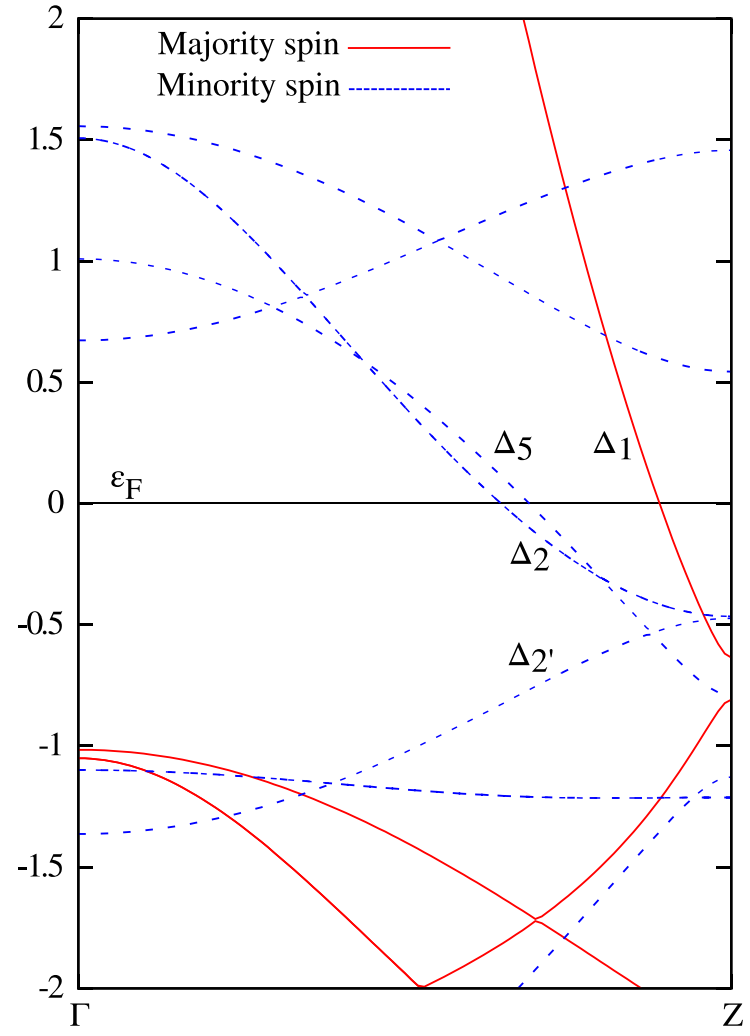

FIG. 3. Computed spin-dependent band structure of CoFe electrode in the [001] direction with respect to the majority [solid line (red)] and minority [dashed line (blue)] spins. The energies are relative to the Fermi energy $\left(\varepsilon_{\mathrm{F}}\right.$ at $\left.0 \mathrm{eV}\right)$.

high variations of the amplitude of TMR, similarly to what was reported previously in the literature [13]. This is in opposition with the early giant magnetoresistance (GMR) studies conducted on metallic multilayer junctions, in which the amplitude of the GMR decays with the increase of the paramagnetic metal layer thicknesses [13]. At the difference of the paramagnetic metal in metallic multijunctions, the $\mathrm{MgO}$ barrier in the MTJs restricts the tunneling current to the channel in the majority spin $\Delta_{1}$ and prevents the occurrence of destructive interferences. Moreover, it is clear that only the available spin channels such as $\Delta_{1}, \Delta_{2}, \Delta_{5}$, originating from the ferromagnetic $\mathrm{CoFe}$ electrodes, are being filtered. The spin channels can be visualized using the spin-dependent band structures of a ferromagnetic CoFe electrode in [001] as shown in Fig. 3. For the majority-spin states of the CoFe electrodes, only the $\Delta_{1}$ states are crossing the Fermi energy, while in case of the minority-spin states, two degenerated $\Delta_{2}$ and $\Delta_{5}$ bands are crossing the Fermi energy (see Fig. 3). Those bands when present at the Fermi level are tunneling through the $\mathrm{MgO}$ barrier and they are filtered depending on their nature. The filtering character of the $\mathrm{MgO}$ barrier for the majority- and minority-spin states is illustrated by the spinand $k$-resolved transmission spectra of the $\mathrm{CoFe}|\mathrm{MgO}| \mathrm{CoFe}$ MTJ stack in Fig. 4. Note that the height and the width of the transmission in the patterns of Fig. 4 are determined by the symmetries of the Bloch wave function tunneling through the barrier.

In the $\mathrm{P}$ configuration, the transmission spectrum of the majority-spin channel is dominated by a broad peak around the center of the two-dimensional Brillouin zone (2D BZ). This is the typical behavior of a coherent tunneling transmission [21] for the $\Delta_{1}$ states at $k_{A}=0$ and $k_{B}=0$ [see Fig. 4(a)].

Switching to the minority-spin channel leads to a strong inhibition of the filtering process that induces spikes at the edges of the 2D BZ [Fig. 4(b)]. That is characteristic for a coherent tunneling process due to the efficient coupling of the $\Delta_{1}$ states of the left and right CoFe electrodes. In the AP configuration, the majority- and minority-spin channels are identical due to the symmetrical electrodes on both sides of the $\mathrm{MgO}$ barrier. Consequently only the majority-spin channel is shown here [see Fig. 4(c)]. The available minority-spin $\Delta_{2}$ and $\Delta_{5}$ states of the left electrode (see Fig. 3) propagate through the $\mathrm{MgO}$ barrier and couple inefficiently with the majority-spin $\Delta_{1}$ state of the right electrode. Subsequently, the transmission of the spin channels in the AP configuration is weaker than in the P configuration and this leads to an enhanced TMR ratio [see Eq. (1)]. The transmission spectra in the P configuration are dominated by broad peaks along the lines $k_{A}=k_{B}=0$ in 2D BZ. Such a pattern is similar to the case of the $\mathrm{Fe}|\mathrm{MgO}| \mathrm{Fe}$ systems [7,21]. This is, however, in contrast with the transmission spectra reported for the $\mathrm{FeCo}|\mathrm{MgO}| \mathrm{FeCo}$ MTJ stack where the Co-O bondings are dominated at the interface between $\mathrm{FeCo}$ and $\mathrm{MgO}$ [8]. In such a configuration the transmission in the AP configuration is concentrated on spin channels at $\left|k_{A}\right|=\left|k_{B}\right|$. Their transmission spectra in the
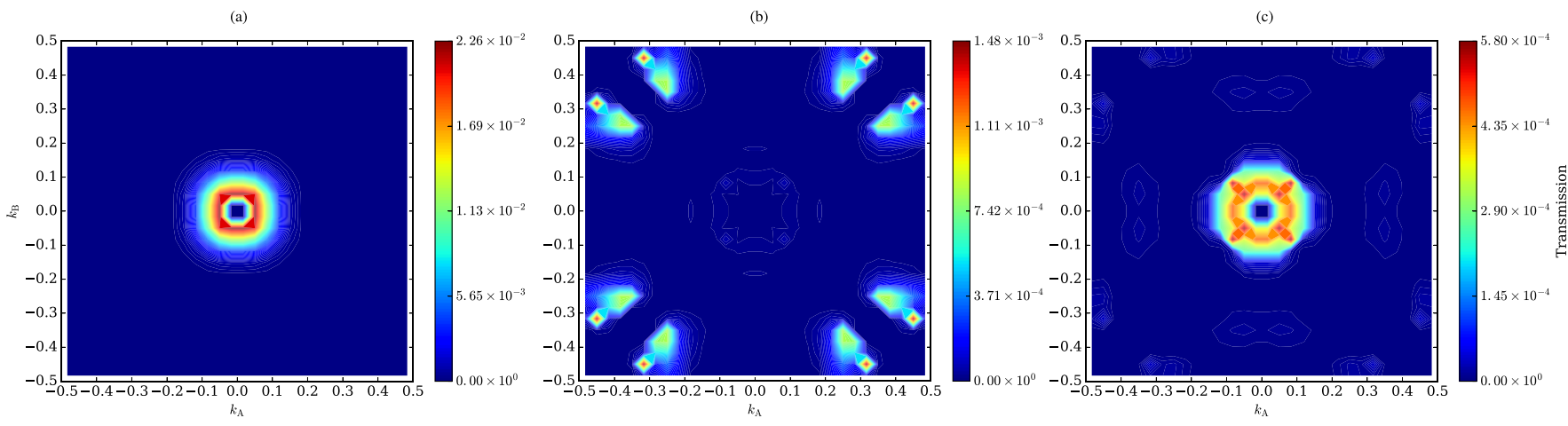

FIG. 4. Spin- and $k$-resolved transmission coefficients of $\mathrm{CoFe}$ (paramagnetic) $\mid \mathrm{CoFe}$ (ferromagnetic) $|\mathrm{MgO}| \mathrm{CoFe}(\mathrm{ferromagnetic}) \mid \mathrm{CoFe}$ (paramagnetic) MTJ stack. The transmission spectra computed for the majority- and minority-spin channels in the parallel configuration are shown in $(a, b)$, while the transmission spectrum computed for the majority-spin channels in the antiparallel configuration is shown in (c). 
AP configuration are hence dominated by peaks along these lines [8]. As reported in Refs. [7,21], in the P configuration, the $\Delta_{1}$ Bloch states tunnel through the $\mathrm{MgO}$ barrier and efficiently couple the electrodes to each other. The increase in the $\mathrm{MgO}$ thickness induces a slow decay rate of the $\Delta_{1}$ states. The transmission probability is therefore at its maximum in the $\mathrm{P}$ configuration. That is in contrast with the AP case, where the $\Delta_{2}, \Delta_{5}$ have a relatively high decay rate in the $\mathrm{MgO}$ barrier due the presence of a large number of nodes. Their decay rates increase further with the thickness of the $\mathrm{MgO}$ barrier as reported in Ref. [22]. Subsequently, the conductance in the $\mathrm{P}$ configuration $\left(G_{\mathrm{P}}\right)$ is dominating the $\mathrm{AP}\left(G_{\mathrm{AP}}\right)$ conductance. This leads to an enhanced TMR ratio [see Eq. (1)]. The overall transmission spectra patterns such as the $\Delta_{1}, \Delta_{2}$, and $\Delta_{5}$ for the majority- and minority-spin states in the $\mathrm{P}$ and AP configurations are not impacted by the change in $\mathrm{CoFe}$ and $\mathrm{MgO}$ thicknesses. However, their transmission probability varies and introduces the oscillatory behavior of the TMR ratio.

The modulations of the electronic properties and the confined wave function induced by quantization effects are not directly reflected in the transmission spectra since there are only subtle changes driven by the film thickness. These are, however, well reflected in the resulting conductances in the $\mathrm{P}$ and AP polarizations used for the calculation of the TMR ratio. Figure 5 summarizes the conductance evolution computed for MTJ stacks having an 11-monolayer-thick MgO barrier in the $\mathrm{P}$ [Fig. 5(a)] and AP [Fig. 5(b)] configurations as a function of the CoFe thickness. The inset figure shows the resulting TMR ratio. Qualitatively, the overall conductances in $\mathrm{P}$ and AP configurations are decreasing with the thickness of CoFe.
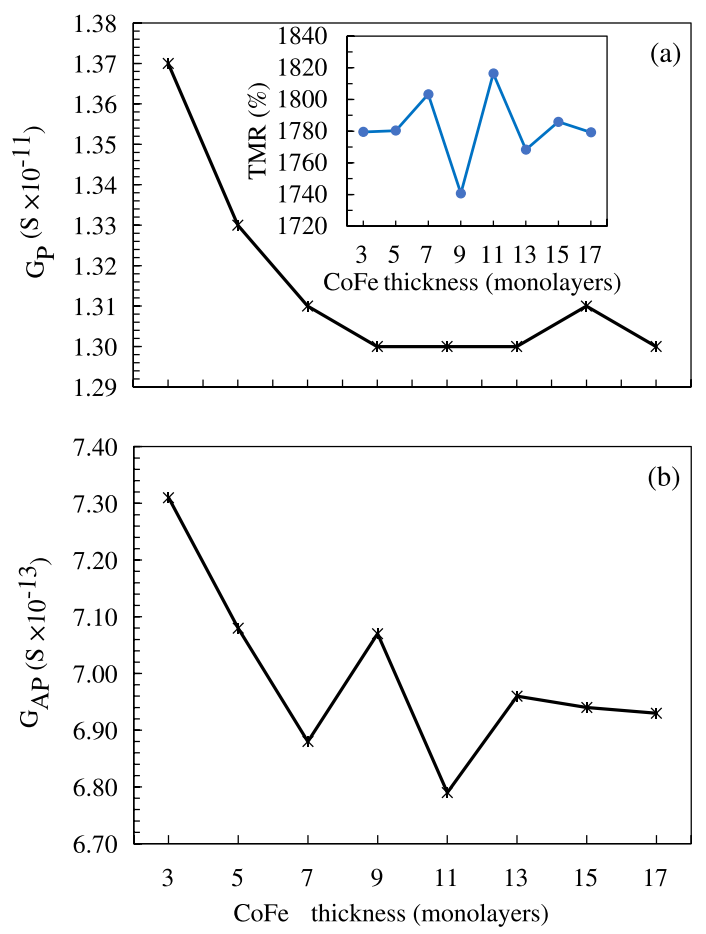

FIG. 5. Computed conductance (in siemens) of a CoFe(paramagnetic) $\mid \mathrm{CoFe}$ (ferromagnetic) $|\mathrm{MgO}| \mathrm{CoFe}$ (ferromagnetic) $\mid \mathrm{CoFe}$ (paramagnetic) MTJ stack in parallel (a) and antiparallel (b) spin configurations as a function of the $\mathrm{CoFe}$ thickness for a $\mathrm{MgO}$ barrier of 11 monolayers thick.
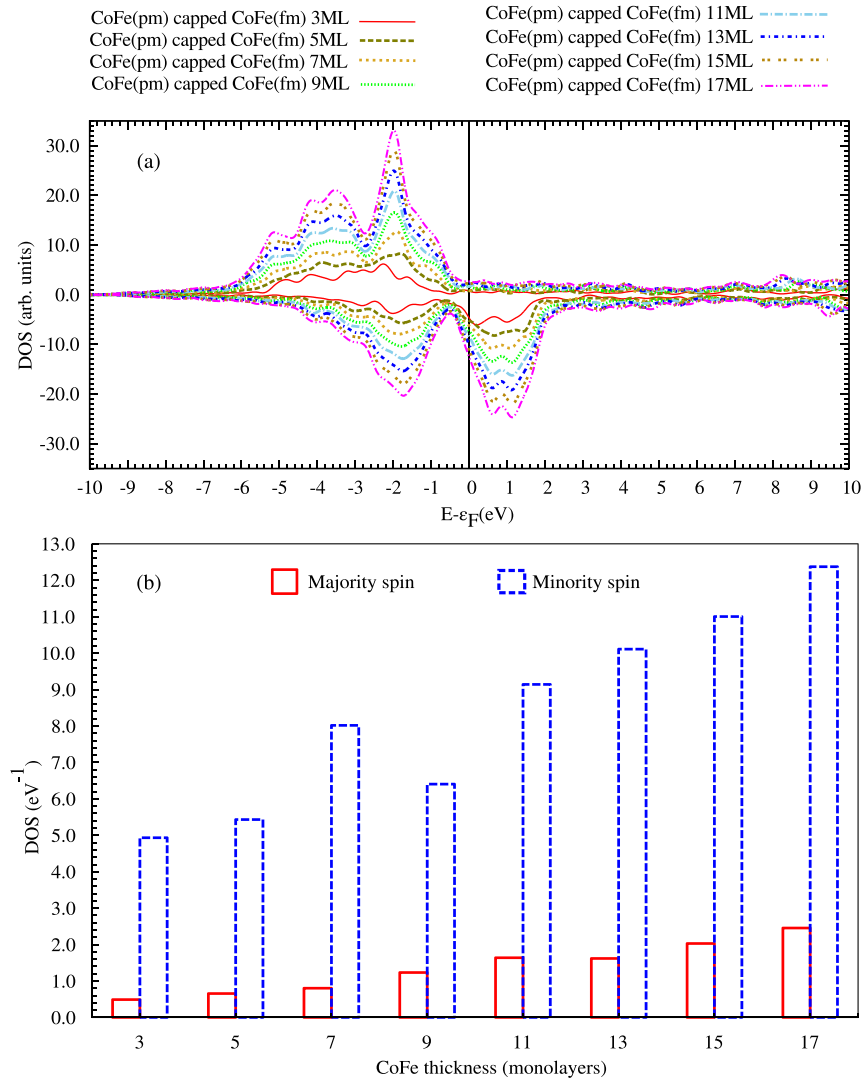

FIG. 6. (a) Computed spin-polarized density of states (DOS) of paramagnetic (pm) CoFe capped ferromagnetic (fm) CoFe in [001] direction for several ferromagnetic $\mathrm{CoFe}$ thicknesses. The vertical line stands for the Fermi level. (b) Computed amplitude of the DOS at the Fermi energy with the majority [solid line (red)] and minority [dashed line (blue)] spins for several ferromagnetic CoFe thicknesses.

$G_{\mathrm{P}}$ saturates above 9 layers, while the $G_{\mathrm{AP}}$ oscillates between 7 and 11 layers of CoFe. An increase of the $G_{\mathrm{AP}}$ is observable at 9 layers of $\mathrm{CoFe}$, while $G_{\mathrm{P}}$ is already saturated, which leads to a decrease in TMR ratio [see inset of Fig. 5(a)]. Our results suggest that the modulation of the electronic properties induced by the confinement of the wave function impacts essentially the transmission probability in the AP configuration [22]. The latter is confirmed by the density of states (DOS) relative to the Fermi energy of the CoFe.

Figure 6 summarizes the evolution of the DOS of the ferromagnetic $\mathrm{CoFe}$ polarizer in the central region at the Fermi level as a function of the thickness (in number of monolayers). The total DOS are shown in Fig. 6(a). The corresponding amplitudes at the Fermi energy are plotted in Fig. 6(b) accounting for the majority (red solid line) and minority (blue dashed line) spins. As expected the amplitude of the DOS increases with the CoFe thickness, which is a typical translation of the thickness-dependent spin frustration effects in the CoFe layer. Note, however, that in case of the minority-spin states, the continuous evolution of the DOS is slightly perturbed for 9 monolayers of $\mathrm{CoFe}$. At that $\mathrm{CoFe}$ thickness, the DOS at the Fermi energy is slightly lower than in the case of 7 monolayers. Consequently, the transmission probability in the AP state is impacted since the minorityspin states are dominated by the $\Delta_{2}$ and $\Delta_{5}$ wave-function 
symmetries. As mentioned above, the confinement of the wave functions essentially impacts the transmission probability in the AP configurations of the ferromagnetic CoFe and hence strongly modulates the TMR ratio. Interestingly, this exercise points towards the fact that a true paramagnetic capping layer such as $\mathrm{Ta}$ in contact with the CoFe ferromagnetic layers should induce or enhance these oscillations.

\section{B. Ta capped MTJ stacks}

In the this section, the impact of the substitution of the $\mathrm{CoFe}$ paramagnetic cap by a Ta cap is investigated. The conductances for the $\mathrm{P}$ and AP states, and the resulting TMR are illustrated in Fig. 7 for the epitaxial MTJ stack with Ta capping layers, now labeled $\mathrm{Ta}(\mathrm{pm})|\mathrm{CoFe}(\mathrm{fm})| \mathrm{MgO}|\mathrm{CoFe}(\mathrm{fm})| \mathrm{Ta}(\mathrm{pm})$. Although the Ta cap follows the $\mathrm{CoFe}$ texture, it differs from the $\mathrm{CoFe}$ by the crystal structure and by the atomic density as described in the methodology section. As a consequence, a different interface is created between the Ta paramagnetic cap and CoFe ferromagnetic electrode. That interface is causing a perturbation to the electronic properties of the $\mathrm{CoFe}$ ferromagnetic layer and it is impacting significantly on the transmission probabilities [Figs. 7(a) and 7(b)]. Although it is hard to quantify the perturbation, we evaluated the difference in the electronic properties of the ferromagnetic $\mathrm{CoFe}$ and consequently the change in the transport properties for the Ta capped and "uncapped" MTJ stacks. We computed for both cases the ferromagnetic $\mathrm{CoFe}$ work functions together with the electron density population analysis at the interfaces. As expected, the conductances in the $\mathrm{P}$ and AP configurations of a perfect epitaxial $\mathrm{Ta}(\mathrm{pm})|\mathrm{CoFe}(\mathrm{fm})| \mathrm{MgO}|\mathrm{CoFe}(\mathrm{fm})| \mathrm{Ta}(\mathrm{pm})$ MTJ evolve with both the $\mathrm{CoFe}$ and the $\mathrm{MgO}$ layer thicknesses. They show not only amplified oscillations but also share common dependencies with respect to the paramagnetic $\mathrm{CoFe}$ case (the "uncapped" MTJ stacks). The TMR has been evaluated for three different $\mathrm{MgO}$ thicknesses (3, 5, and 11 monolayers) as illustrated in Fig. 7(c). Similarly to the previous stacks (see Fig. 2), the TMR increases with the $\mathrm{MgO}$ thickness due to the enhanced filtering of the Bloch states with respect to certain symmetries of the wave function. This behavior is inherent to the $\mathrm{MgO}$ barrier and is independent of the nature of the capping materials Refs. [7,21]. On the contrary, the amplitude of the TMR significantly varies with the thickness of the CoFe ferromagnetic layers when comparing it to similar MTJ stacks without a capping layer [see Figs. 7(c) and 2)]. Interestingly, our simulations suggest that the amplitude of the TMR is slightly higher for certain thicknesses of $\mathrm{CoFe}$ and $\mathrm{MgO}$ for the case of the "Ta capped MTJ stacks" when compared to the uncapped ones. These fluctuations are bound to the modulation of the electronic properties of the CoFe polarizer induced by the Ta capping layer. The analysis of the conductances [Figs. 7(a) and 7(b)] displays pronounced oscillations of the conductances when the layer thickness of both the ferromagnetic $\mathrm{CoFe}$ and $\mathrm{MgO}$ increases. This is illustrated by the comparison of the DOS of the CoFe with the Ta cap and with the paramagnetic CoFe cap (Fig. 8). For the sake of conciseness, we report only the DOS of 5 and 13 monolayers CoFe for both $\mathrm{Ta}(\mathrm{pm}) \mid \mathrm{CoFe}(\mathrm{fm})$ and $\mathrm{CoFe}(\mathrm{pm}) \mid \mathrm{CoFe}(\mathrm{fm})$ capped MTJ stacks [Fig. 8(a)]. The amplitude of the DOS at the Fermi energy is plotted in
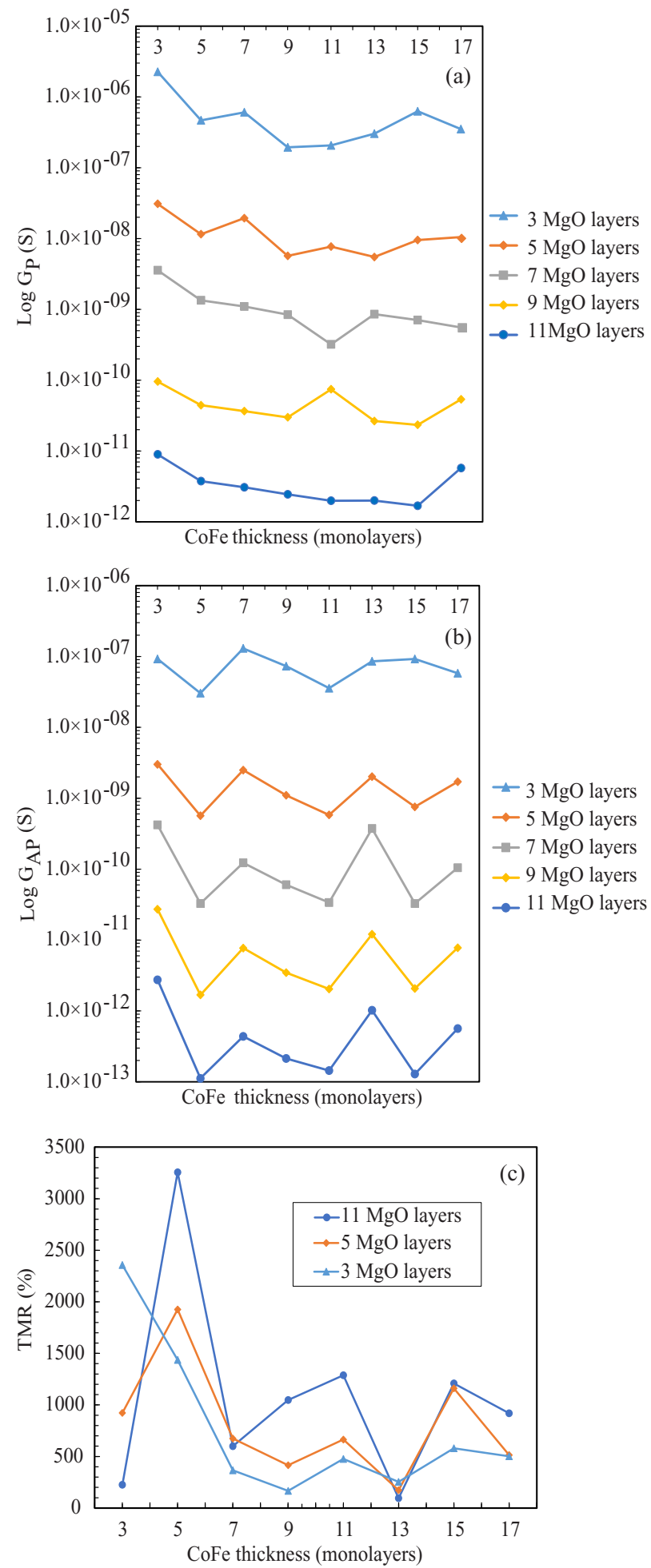

FIG. 7. Evolution of the computed conductance in parallel (a) and antiparallel (b) states of $\mathrm{Ta}$ (paramagnetic) $\mid \mathrm{CoFe}$ (ferromagnetic) $\mid$ $\mathrm{MgO} \mid \mathrm{CoFe}$ (ferromagnetic)|Ta(paramagnetic) MTJ stack as a function of the $\mathrm{CoFe}$ thickness for different $\mathrm{MgO}$ barrier thicknesses. (c) is the evolution of the computed TMR ratio for 3, 5, and 11 monolayers of $\mathrm{MgO}$ as a function of CoFe thickness.

Fig. 8(b) as a function of the ferromagnetic CoFe thickness. Interestingly, the insertion of the Ta cap shifts the Fermi energy of CoFe polarizer due to the impact of the Ta cap on the work 

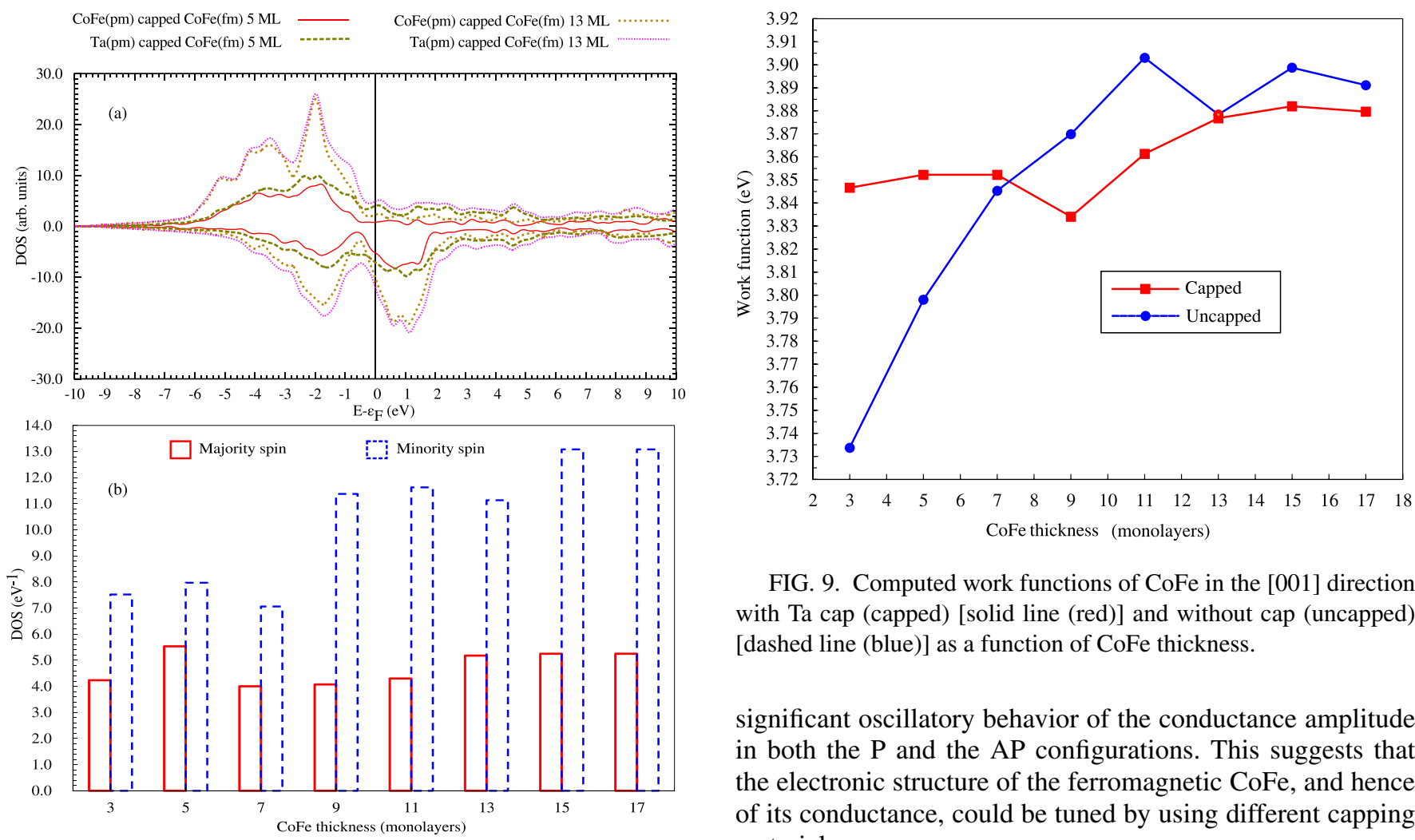

FIG. 9. Computed work functions of CoFe in the [001] direction with Ta cap (capped) [solid line (red)] and without cap (uncapped) [dashed line (blue)] as a function of CoFe thickness.

significant oscillatory behavior of the conductance amplitude in both the $\mathrm{P}$ and the AP configurations. This suggests that the electronic structure of the ferromagnetic $\mathrm{CoFe}$, and hence of its conductance, could be tuned by using different capping materials.

The electronic transport properties depend not only on the

FIG. 8. (a) Computed spin-polarized density of states (DOS) of paramagnetic (pm) Ta capped ferromagnetic (fm) $\mathrm{CoFe}$ in [001] for ferromagnetic $\mathrm{CoFe}$ of 5 and 13 monolayers thick (and compared with the respective paramagnetic $\mathrm{CoFe}$ cap cases). The vertical line stands for the Fermi level. (b) Computed amplitude of the DOS at the Fermi energy with the majority [solid line (red)] and minority [dashed line (blue)] spins for several ferromagnetic CoFe thicknesses.

function. To gain some insights on how the latter evolves, we evaluated the work function of an ideal $\mathrm{Ta} \mid \mathrm{CoFe}$ slab surrounded by vacuum. The dimension of the unit cell along the vacuum direction has been set to minimize the presence of any spurious electric field induced by the asymmetry of the slab. Figure 9 shows the resulting work function fluctuations of ferromagnetic $\mathrm{CoFe}$ with and without Ta cap as a function of different $\mathrm{CoFe}$ thicknesses. The impact of the confinement of the wave functions and the CoFe thickness increase is translated by the work function fluctuations of uncapped $\mathrm{CoFe}$. The work function of CoFe varies from about 3.7 to $3.9 \mathrm{eV}$ for thicknesses of CoFe ranging from 3 to 17 layers (see Fig. 9). In the presence of $\mathrm{Ta}$, the work function of $\mathrm{CoFe}$ is less modulated (see Fig. 9). That can be attributed to the fcc crystal structure being imposed on $\mathrm{Ta}$, which favors the delocalization of the wave function of $\mathrm{CoFe}$ (thanks to its symmetry). As a result, it activates not only the $\Delta_{1}, \Delta_{2}$, and $\Delta_{5}$ bands but also some additional channels in the tunneling process. That is reflected in the increased intensity of the DOS for the Ta capped CoFe case when compared to the uncapped case [see Figs. 8(a) and 8(b)]. Those changes in terms of electronic structure directly impact on the transmission probabilities [see Figs. 7(a) and 7(b)]. The combination of the wave function confinement in $\mathrm{CoFe}$ and of the shift of the Fermi level due to the presence of Ta induces a intrinsic confinement of the wave function (and hence on the resulting electronic structure of the $\mathrm{CoFe}$ and $\mathrm{MgO}$ layers) but also on the electronic properties of the $\mathrm{Ta}(\mathrm{pm}) \mid \mathrm{CoFe}(\mathrm{fm})$ and $\mathrm{CoFe}(\mathrm{fm}) \mid \mathrm{MgO}$ interfaces. The electronic properties of an interface between two materials are governed by the nature of the bonds present and of their charge transfer, which also set the electrostatic alignment between the materials (in other words, the band offset). The band offset hence reflects the interface dipole induced by the difference in electronegativity between the materials. That difference occurs naturally upon the formation of bonds. This interfacial charge transfer can be quantified by an electronic density population analysis such as the one proposed by Mülliken [23]. Although qualitative, this analysis (see Fig. 10) illustrates the presence of a charge excess/depletion at the interface with respect to the bulk value. Figures 10(a) and 10(b) show the electronic population analysis for the $\mathrm{CoFe}(\mathrm{pm})$ and the $\mathrm{Ta}(\mathrm{pm})$ capped MTJ stack composed of five atomic planes of ferromagnetic $\mathrm{CoFe}$ and $\mathrm{MgO}$. The interfacial charge transfer is rather confined to the first two monolayers at the interface and is quickly screened in the metal. Depending on the material polarizability, the chemical heterogeneity of the film contributes to the propagation of the changes in charge transfer. At the $\mathrm{CoFe} \mid \mathrm{MgO}$ interfaces, the charge transfer from the first to the third monolayer is relatively important due to the presence of $\mathrm{CoFe}(\mathrm{pm})$ and $\mathrm{Ta}(\mathrm{pm})$ caps [see Figs. 10(a) and 10(b)]. The first Fe monolayer in contact with $\mathrm{MgO}$ shows a depleted electron density due to the contact with electronegative $\mathrm{O}$ atom. This depletion generates charge images within $\mathrm{CoFe}$ and the charge transfer is minimized by a weaker electron gain of the second Co layer. As a consequence, a net electron transfer occurs from the $\mathrm{CoFe}$ polarizer to the insulating $\mathrm{MgO}$. This is consistent 

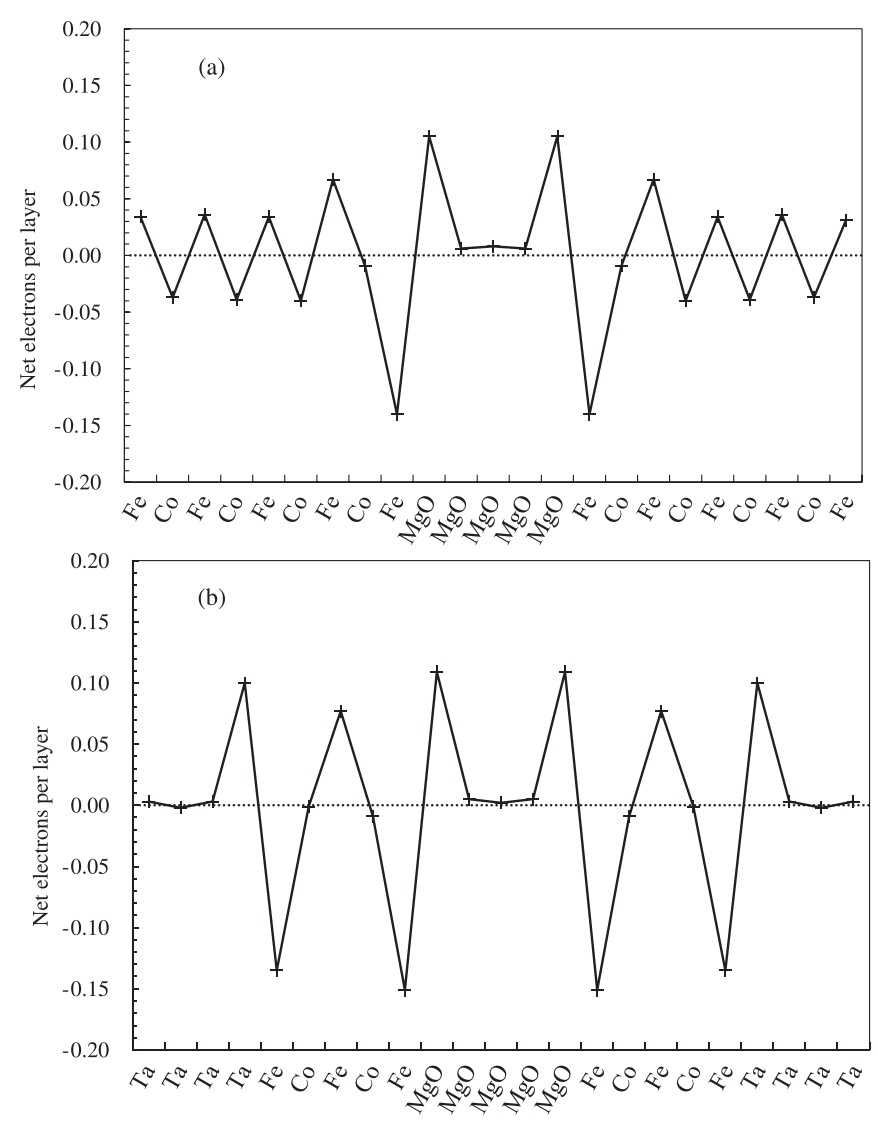

FIG. 10. Computed charge transfer (in electron units) at the $\mathrm{CoFe}$ (a) and $\mathrm{Ta}$ (b) paramagnetic capped (4 monolayers thick) $\mathrm{CoFe}$ (ferromagnetic) $|\mathrm{MgO}| \mathrm{CoFe}$ (ferromagnetic) MTJ stack (5 monolayers thick of $\mathrm{CoFe}$ and $\mathrm{MgO}$ ) interfaces, as extracted from the Mülliken population analysis. The positive and negative net electron per monolayer stand for a gain and a loss (depletion) of electron density, respectively.

with previously reported studies in $\mathrm{Fe} \mid \mathrm{MgO}$ and $\mathrm{Co} \mid \mathrm{MgO}$ MTJs [7,8] and is a $\mathrm{CoFe} \mid \mathrm{MgO}$ specific interface related property. At the $\mathrm{CoFe}(\mathrm{pm}) \mid \mathrm{CoFe}(\mathrm{fm})$ interfaces, the similarity in chemical composition is such that no distinct interfacial charge transfer occurs. In contrast, the difference in electronegativity between $\mathrm{CoFe}$ and $\mathrm{Ta}$ is such that an additional charge transfer occurs at the interface between $\mathrm{Ta}(\mathrm{pm}) \mid \mathrm{CoFe}(\mathrm{fm})$ interfaces [Fig. 10(b)]. Although the former is limited to the first Ta layer, it contributes to the electron depletion of the ferromagnetic layer [see Fig. 10(b)], independently of the thickness of the ferromagnetic $\mathrm{CoFe}$ and $\mathrm{MgO}$. The resulting dipole layer has a direct impact on the interface electrostatics and hence on the band alignments. The electrostatic difference potentials are estimated for the $\mathrm{Ta}(\mathrm{pm})$ cap and uncapped MTJ stacks. The electrostatic potential difference is calculated from the electron density difference which is the difference between the self-consistent valence charge density and the superposition of atomic valence density. Once aligning these electrostatic potential differences, the resulting shift due to the Ta cap layer is found to be $0.88 \mathrm{eV}$.

At this stage, it is interesting to compare the evolution of the TMR for the $\mathrm{Ta}(\mathrm{pm})|\mathrm{CoFe}(\mathrm{fm})| \mathrm{MgO}|\mathrm{CoFe}(\mathrm{fm})| \mathrm{Ta}(\mathrm{pm})$ capped and the $\mathrm{CoFe}(\mathrm{pm})|\mathrm{CoFe}(\mathrm{fm})| \mathrm{MgO}|\mathrm{CoFe}(\mathrm{fm})| \mathrm{Ta}(\mathrm{pm})$ uncapped MTJ stacks (see Figs. 2 and 7). At a given barrier thickness, the TMR ratio oscillates more significantly in the case of the Ta capped MTJ than the case of the uncapped MTJ. That leads to an enhancement of the TMR for thin ferromagnetic $\mathrm{CoFe}$ layers. This phenomenon arises from the combination of the wave function confinement in the $\mathrm{CoFe}$ layer and the shift in band offset due to the interface dipole. The former is amplified in the case of thin ferromagnetic CoFe layers in contact with the Ta cap due to the breaking of both the crystal continuity and atomic density. As a result, the DOS of the $d$ bands and the work function of $\mathrm{CoFe}$ defining the Fermi level get strongly impacted by the variations of the ferromagnetic CoFe thicknesses (see Figs. 8 and 9). Furthermore, the crystal symmetry of the capping layers plays an important role in the transmission, as also illustrated by the environment dependence in Refs. [5,12].

The spin- and $k$-resolved transmission coefficients at the Fermi energy for the Ta capped MTJ stacks are shown in Fig. 11 for the case of 5 and 13 layers of ferromagnetic CoFe. They illustrate how the transport spin channels are affected by both the Ta cap and the variations of the ferromagnetic CoFe thicknesses. The introduction of Ta as a cap leads to significant changes in the transmission of the majority-spin states in the parallel configuration due to a strong suppression of the transmission channel in the middle of the 2D BZ. That is in contrast with the TMR spectra of the $\mathrm{CoFe}(\mathrm{pm})$ uncapped MTJ stacks, where the $\Delta_{1}$ band is strongly contributing to the transmission at the 2D BZ center [see Fig. 4(a)], since it is crossing the Fermi energy in the [001] direction of $\mathrm{CoFe}$ (see Fig. 3). In the presence of Ta, the absence of the $\Delta_{1}$ Bloch wave functions points towards a shift of the Fermi level due to the interface dipole/change in work function, as detailed in the section above. As a consequence, some other complex Bloch wave functions are activated at the interface of the $\mathrm{MgO}$ barrier and these are present in the transmission spectrum. The second notable observation is about the AP state. The majority and minority spins have significantly different signatures, whereas they are identical in absence of a Ta cap. This stresses the important role of the Ta cap on the transmission spin channels. Moreover, the transmission spectra of the majority and minority spins are not identical in the AP state. Consequently, the transmitted spin channels are different. There is a relative increase in the amplitude of the transmission in all the configurations of minority-spin states at the edges of the 2D BZ, which we attribute to the contribution of the $\Delta_{2}, \Delta_{2^{\prime}}$, and $\Delta_{5}$ bands to the transmission [8].

It is also worth stressing that there is a significant difference in the transmission coefficients patterns for the 5 and 13 layers of ferromagnetic $\mathrm{CoFe}$ in the presence of $\mathrm{Ta}$ cap, as shown in Fig. 11 (from top to bottom panels). In the P configuration, the transmission coefficients are larger in the case of the 5 monolayers (top panels) than in the case for the 13 monolayers (bottom panels). In contrast, the transmission coefficient spectra of the Ta capped MTJ with CoFe of 13 monolayers thick (bottom panels in Fig. 11) show a higher tunneling probability in the AP configuration than those of the Ta capped MTJ with 5 monolayers $\mathrm{CoFe}$ (top panels in Fig. 11). Consequently, the thin CoFe polarizer in contact with Ta yields a larger TMR ratio. A similar effect is obtained for Ta capped MTJ stacks with different $\mathrm{MgO}$ and CoFe thicknesses (not shown here). 

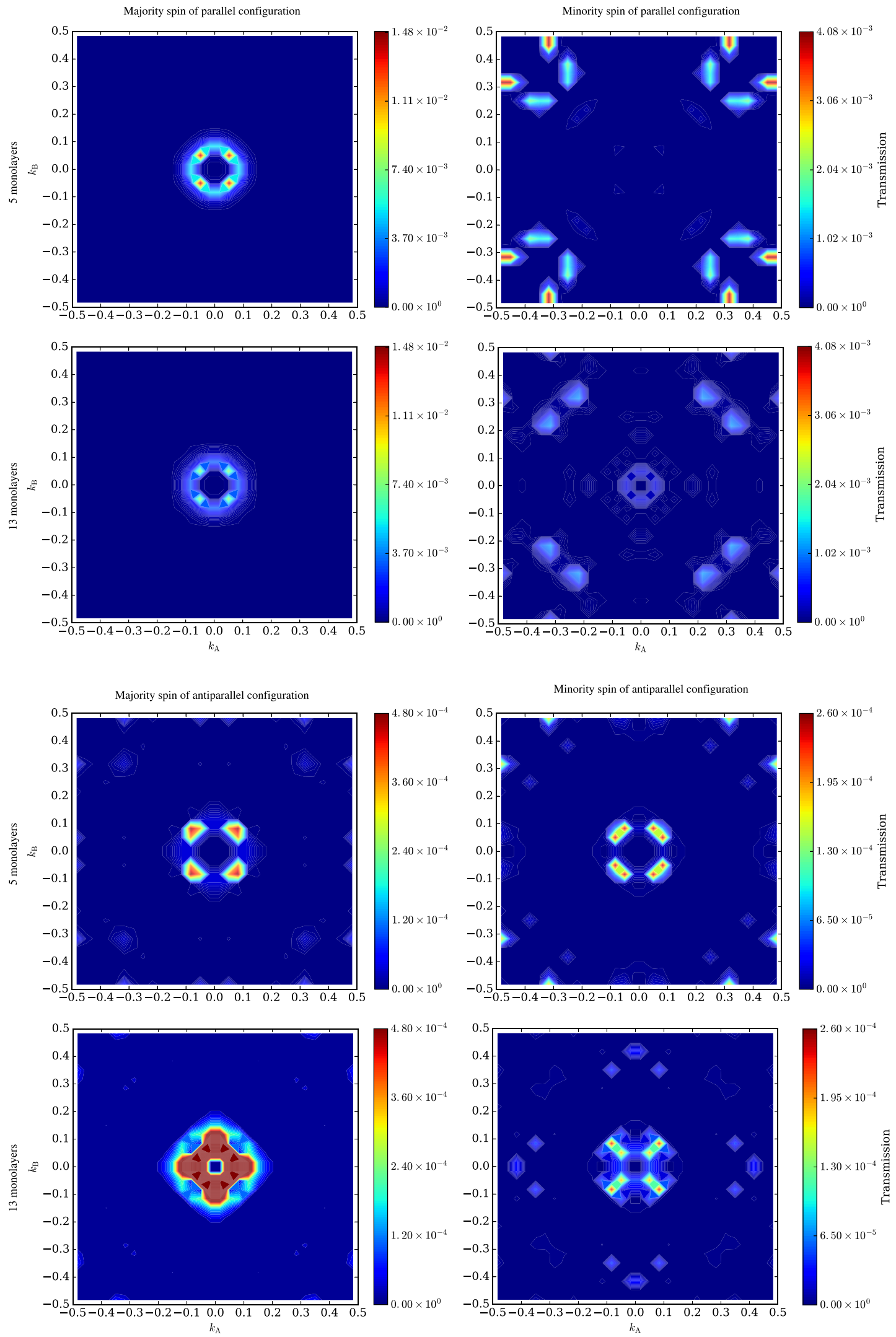

FIG. 11. Computed spin- and $k$-resolved transmission coefficients of $\mathrm{Ta}$ (paramagnetic) $\mid \mathrm{CoFe}$ (ferromagnetic) $|\mathrm{MgO}| \mathrm{CoFe}($ ferromagnetic) $\mid$ $\mathrm{Ta}$ (paramagnetic) MTJ stacks. The 4 panels on the left and right sides correspond to the parallel configuration of minority and majority spins, respectively, for ferromagnetic $\mathrm{CoFe}$ of 5 and 13 monolayers thick with $\mathrm{MgO}$ thickness of 5 monolayers. The bottom 4 panels on the left and right sides correspond to the antiparallel configuration of minority and majority spins, respectively, for ferromagnetic CoFe of 5 and 13 monolayers thick with $\mathrm{MgO}$ thickness of 5 monolayers. 


\section{CONCLUSIONS}

First-principles simulations suggest that the TMR in MTJ stacks is sensitive to both the nature of the capping layers in contact with $\mathrm{CoFe}$ and to the $\mathrm{CoFe}$ and $\mathrm{MgO}$ thicknesses as reported experimentally $[15,16]$. The impact of the capping metal has been analyzed for the case of Ta, for which the presence of a dipole at the $\mathrm{Ta} \mid \mathrm{CoFe}$ interface leads to significant changes in the computed TMR. As a result, the amplitude of the oscillations is strongly enhanced with respect to the amplitude reported experimentally for similar MTJ stacks [5,12]. The origin of this difference is still unclear [24]. Aside from the theoretical intrinsic limits of DFT and of the accuracy of the description of the electronic properties, it can also be attributed to the presence of the artificial ideal epitaxial atomic layers used in the MTJ stack models to compute the TMR. As importantly, the presence of interfacial roughness, oxidation, intermixing, oxygen vacancies in the $\mathrm{MgO}$ barrier, and eventually of diffusion [24] in the MTJ stacks grown experimentally can explain the difference. Set within these limitations, this theoretical work suggests that, in the range of experimental thicknesses of the MTJ stack used in the fabrication of the STT switching based MRAM, the Ta capping metal impacts the TMR ratio and is beneficial for the device performance. It is interesting to stress that the interface dipole/offset tuning depends on the nature of the metal used, as already demonstrated by the engineering of work functions in metal-oxide-semiconductor field-effect transistor (MOSFET) devices [25].

\section{ACKNOWLEDGMENTS}

This work is a part of the imec industrial affiliations program (IIAP) on nonvolatile memories. The authors thank Dr. G. S. Kar and Dr. A. Furnemont for the management of the research program.
TABLE I. Atomic coordinates (in angstroms) of the paramagnetic CoFe and Ta capped MTJ stacks.

\begin{tabular}{|c|c|c|c|c|c|c|c|}
\hline \multicolumn{4}{|c|}{ CoFe cap } & \multicolumn{4}{|c|}{ Ta cap } \\
\hline Element & $x$ & $y$ & $z$ & Element & $x$ & $y$ & $z$ \\
\hline $\mathrm{Fe}$ & 0.000 & 0.000 & 0.62 & $\mathrm{Ta}$ & 0.000 & 0.000 & 1.1785 \\
\hline $\mathrm{Co}$ & 0.000 & 1.465 & 2.018 & $\mathrm{Ta}$ & 0.000 & 1.465 & 3.5355 \\
\hline $\mathrm{Fe}$ & 0.000 & 0.000 & 3.258 & $\mathrm{Ta}$ & 0.000 & 0.000 & 5.8925 \\
\hline Co & 0.000 & 1.465 & 4.656 & $\mathrm{Ta}$ & 0.000 & 1.465 & 8.2495 \\
\hline $\mathrm{Fe}$ & 0.000 & 0.000 & 5.896 & $\mathrm{Fe}$ & 0.000 & 0.000 & 10.6585 \\
\hline $\mathrm{Co}$ & 0.000 & 1.465 & 7.294 & Co & 0.000 & 1.465 & 11.8985 \\
\hline $\mathrm{Fe}$ & 0.000 & 0.000 & 8.534 & $\mathrm{Fe}$ & 0.000 & 0.000 & 13.2965 \\
\hline $\mathrm{Mg}$ & 0.000 & 1.465 & 10.634 & $\mathrm{Mg}$ & 0.000 & 1.465 & 15.3965 \\
\hline $\mathrm{Mg}$ & 0.000 & 0.000 & 12.733 & $\mathrm{Mg}$ & 0.000 & 0.000 & 17.4955 \\
\hline $\mathrm{Mg}$ & 0.000 & 1.465 & 14.832 & $\mathrm{Mg}$ & 0.000 & 1.465 & 19.5945 \\
\hline $\mathrm{O}^{\circ}$ & 0.000 & 0.000 & 10.634 & $\mathrm{O}$ & 0.000 & 0.000 & 15.3965 \\
\hline $\mathrm{O}$ & 0.000 & 1.465 & 12.733 & $\mathrm{O}$ & 0.000 & 1.465 & 17.4955 \\
\hline $\mathrm{O}$ & 0.000 & 0.000 & 14.832 & $\mathrm{O}$ & 0.000 & 0.000 & 19.5945 \\
\hline $\mathrm{Fe}$ & 0.000 & 00.00 & 16.932 & $\mathrm{Fe}$ & 0.000 & 00.00 & 21.6945 \\
\hline Co & 0.000 & 1.465 & 18.172 & Co & 0.000 & 1.465 & 22.9345 \\
\hline $\mathrm{Fe}$ & 0.000 & 0.000 & 19.57 & $\mathrm{Fe}$ & 0.000 & 0.000 & 24.3325 \\
\hline Co & 0.000 & 1.465 & 20.81 & $\mathrm{Ta}$ & 0.000 & 1.465 & 26.7415 \\
\hline $\mathrm{Fe}$ & 0.000 & 0.000 & 22.208 & $\mathrm{Ta}$ & 0.000 & 0.000 & 29.0985 \\
\hline $\mathrm{Co}$ & 0.000 & 1.465 & 23.448 & $\mathrm{Ta}$ & 0.000 & 1.465 & 31.4555 \\
\hline $\mathrm{Fe}$ & 0.000 & 0.000 & 24.846 & $\mathrm{Ta}$ & 0.000 & 0.000 & 33.8125 \\
\hline
\end{tabular}

\section{APPENDIX}

The atomic coordinates (in angstrom) of the paramagnetic CoFe capped MTJ stacks [CoFe(paramagnetic) $\mid \mathrm{CoFe}$ (ferromagnetic) $|\mathrm{MgO}| \mathrm{CoFe}$ (ferromagnetic) $\mid \mathrm{CoFe}$ (paramagnetic)] are listed on the left side of Table I while those of the Ta capped MTJ stack [Ta(paramagnetic) $\mid \mathrm{CoFe}$ (ferromagnetic)| $\mathrm{MgO} \mid \mathrm{CoFe}$ (ferromagnetic) $\mid \mathrm{Ta}$ (paramagnetic)] are on the right side of the table. The MTJ stack in the two models is composed of three monolayers of $\mathrm{CoFe}$ and $\mathrm{MgO}$, while their capping layer is 4 monolayers thick.
[1] M. Jullière, Phys. Rev. Lett. A 54, 225 (1975).

[2] W. J. Gallagher and S. S. Parkin, IBM J. Res. Dev. 50, 5 (2006).

[3] W. Butler, Sci. Technol. Adv. Mater. 9, 225B (2008).

[4] J. S. Moodera, L. R. Kinder, T. M. Wong, and R. Meservey, Phys. Rev. Lett. 74, 3273 (1995).

[5] Y. M. Lee, J. Hayakawa, S. Ikeda, F. Matsukura, and H. Ohno, Appl. Phys. Lett. 90, 212507 (2007).

[6] J. M. Teixeira, J. Ventura, J. P. Araujo, J. B. Sousa, P. Wisnioki, and P. P. Freitas, Appl. Phys. Lett. 96, 262506 (2010).

[7] W. H. Butler, X.-G. Zhang, T. C. Schulthess, and J. M. MacLaren, Phys. Rev. B 63, 054416 (2001).

[8] X.-G. Zhang and W. H. Butler, Phys. Rev. B 70, 172407 (2004).

[9] F. Bonell, T. Hauet, S. Andrieu, F. Bertran, P. Le Fèvre, L. Calmels, A. Tejeda, F. Montaigne, B. Warot-Fonrose, B. Belhadji, A. Nicolaou, and A. Taleb-Ibrahimi, Phys. Rev. Lett. 108, 176602 (2012).
[10] A. V. Khvalkovskiy, D. Apalkov, S. Watts, R. Chepulskii, R. S. Beach, A. Ong, X. Tang, A. Driskill-Smith, W. H. Butler, P. B. Visscher, D. Lottis, E. Chen, V. Nikitin, and M. Krounbi, J. Phys. D: Appl. Phys. 46, 074001 (2013).

[11] H. X. Yang, M. Chshiev, B. Dieny, J. H. Lee, A. Manchon, and K. H. Shin, Phys. Rev. B 84, 054401 (2011).

[12] C. Heiliger, M. Gradhand, P. Zhan, and I. Mertig, Phys. Rev. Lett. 99, 066804 (2007).

[13] G. Autès, J. Mathon, and A. Umerski, Phys. Rev. B 84, 134404 (2011).

[14] J. Swerts, S. Mertens, T. Lin, S. Couet, Y. Tomczak, K. Sankaran, G. Pourtois, W. Kim, J. Meersschaut, L. Souriau, D. Radisic, S. Van Elshocht, G. Kar, and A. Furnemont, Appl. Phys. Lett. 106, 262407 (2015).

[15] Y. Shiota, F. Bonell, S. Miwa, N. Mizuochi, T. Shinjo, and Y. Suzuki, Appl. Phys. Lett. 103, 082410 (2013).

[16] A. Rajanikanth, T. Hauet, F. Montaigne, S. Mangin, and S. Andrieu, Appl. Phys. Lett. 103, 062402 (2013). 
[17] H. X. Yang, M. Chshiev, A. Kalitsov, A. Schuhl, and W. H. Butler, Appl. Phys. Lett. 96, 262509 (2010).

[18] W. C. Tsai, S. C. Liao, H. C. Hou, C. T. Yen, Y. H. Wang, H. M. Tsai, F. H. Chang, H. J. Lin, and C.-H. Lai, Appl. Phys. Lett. 100, 172414 (2012).

[19] M. Brandbyge, J.-L. Mozos, P. Ordejón, J. Taylor, and K. Stokbro, Phys. Rev. B. 65, 165401 (2002).

[20] J. P. Perdew, K. Burke, and M. Ernzerhof, Phys. Rev. Lett. 77, 3865 (1996).
[21] M. Stilling, K. Stokbro, and K. Flensberg, Mol. Simul. 33, 557 (2007).

[22] M. Stilling, K. Stokbro, and K. Flensberg, J. Comput. Aided Mater. Des. 14, 141 (2007).

[23] R. S. Mulliken, J. Chem. Phys. 23, 1833 (1955).

[24] Z. Bai, L. Shen, Q. Wu, M. Zeng, and J.-S. Wang, Phys. Rev. B 87, 014114 (2013).

[25] J. J. Paggel, C. M. Wei, M. Y. Chou, D.-A. Luh, T. Miller, and T.-C. Chiang, Phys. Rev. B 66, 233403 (2002). 